Article

\title{
Efficient Arrangement of the Replication Fork Trap for In Vitro Propagation of Monomeric Circular DNA in the Chromosome-Replication Cycle Reaction
}

\author{
Tomonori Hasebe, Kouhei Narita, Shiomi Hidaka and Masayuki Su'etsugu * \\ Department of Life Science, College of Science, Rikkyo University, 3-34-1 Nishi-Ikebukuro, Toshima-ku, Tokyo \\ 171-8501, Japan; 18LD012D@rikkyo.ac.jp (T.H.); grampus.13.k.n@gmail.com (K.N.); \\ s-hidaka@labo.med.osaka-u.ac.jp (S.H.) \\ * Correspondence: suetsugu@rikkyo.ac.jp; Tel./Fax: +81-3-3985-2372
}

Received: 4 September 2018; Accepted: 23 September 2018; Published: 25 September 2018

\begin{abstract}
Propagation of genetic information is a fundamental prerequisite for living cells. We recently developed the replication cycle reaction (RCR), an in vitro reaction for circular DNA propagation, by reconstitution of the replication cycle of the Escherichia coli chromosome. In RCR, two replication forks proceed bidirectionally from the replication origin, oriC, and meet at a region opposite oriC, yielding two copies of circular DNA. Although RCR essentially propagates supercoiled monomers, concatemer byproducts are also produced due to inefficient termination of the replication fork progression. Here, we examined the effect of the Tus-ter replication fork trap in RCR. Unexpectedly, when the fork traps were placed opposite oriC, mimicking their arrangement on the chromosome, the propagation of circular DNA was inhibited. On the other hand, fork traps flanking oriC allowed efficient propagation of circular DNA and repressed concatemer production. These findings suggest that collision of the two convergence forks through the fork trap is detrimental to repetition of the replication cycle. We further demonstrate that this detrimental effect was rescued by the UvrD helicase. These results provide insights into the way in which circular DNA monomers replicate repetitively without generating concatemers.
\end{abstract}

Keywords: DNA amplification; chromosome replication; replication fork; replication termination

\section{Introduction}

Self-replication is a fundamental property of living systems inherited from the origin of life. To achieve self-replication, cells must replicate their genetic information. In vitro reconstruction is a powerful approach to understand the self-replication event [1,2]. Although polymerase chain reaction (PCR) is an enabling technology to propagate genetic information in vitro through a repetitive cycle of DNA replication, it requires artificial thermal cycling at high temperatures. On the other hand, living cells can continuously propagate large-sized genomic DNA under isothermal conditions through an enzymatic repetition of replication cycles involving large numbers of proteins.

In Escherichia coli, replication of the circular chromosome starts from a single replication origin, oriC. Two replication forks proceed bidirectionally from oriC and eventually merge in a chromosome terminus region that is located approximately opposite oriC on the circular map, thereby generating two copies of the circular chromosome [3,4]. A series of replication reactions have been reconstituted in vitro using purified proteins and a circular DNA containing oriC $[5,6]$. The DnaA protein binds to oriC and initiates replication by unwinding the DNA duplex $[7,8]$. DnaB helicase then expands the unwound region to allow association of the replication fork machinery, including single-stranded DNA-binding protein (SSB), DnaG primase, and DNA polymerase III holoenzyme $[9,10]$, the latter 
of which synthesizes the leading and lagging strand concurrently. Bidirectional progression of two replication forks from oriC has been demonstrated in the reconstituted replication system [11-14].

To control termination of replication, the E. coli chromosome (4.6 Mb) contains ten ter (termination) sites within the $2 \mathrm{Mb}$ region opposite oriC [15-17]. Tus binds to ter and blocks replication fork progression in an orientation-dependent manner [18-21]. The chromosomal orientation of ter sites is arranged to allow replication forks from oriC to enter, but not exit, the termination region. Although the Tus-ter fork trap can restrict forks travelling in the ter-to oriC direction, it does not appear to be directly involved in the completion of replication because tus-deficient cells complete chromosome replication and grow normally [22]. Replication fork arrest by the Tus-ter system has been demonstrated in the reconstituted oriC replication system using a circular DNA containing ter sites opposite oriC [11,12,14]; however, the effect of the fork trap on repetition of the replication cycle remains unclear.

We recently developed the replication cycle reaction (RCR) by in vitro reconstitution of the E. coli chromosome-replication cycle. RCR consists of replication initiation at oriC, bidirectional progression of replication forks, completion of replication, and segregation of two daughter circular DNA molecules [23]. The segregation process produces monomeric circular DNA that is topologically identical to the input template DNA, thus allowing autonomous repetition of the replication cycle under isothermal conditions. RCR can propagate circular DNA exponentially with a doubling time of approximately $8 \mathrm{~min}$, even from a single DNA molecule. Because RCR uses the chromosome-replication system, large DNAs (longer than $200 \mathrm{~kb}$ ) can be propagated as intact circular DNA molecules, and the replication fidelity is extremely high (approximately $1.2 \times 10^{-8}$ errors per base per cycle) [23]. Although RCR produces supercoiled monomer molecules, larger-sized concatemer molecules are partially produced due to rolling circle replication caused by inefficient termination of the replication fork progression.

Here, we investigated the use of the Tus-ter fork trap to specifically prevent the production of unwanted concatemer DNA molecules in RCR. We found that fork traps positioned opposite oriC, mimicking their arrangement on the chromosome map, severely inhibited DNA propagation. By contrast, positioning of fork traps at a region flanking oriC, rather than the termination site, allowed DNA propagation without concatemer formation. Furthermore, using UvrD helicase in RCR eliminated the inhibitory effect of the fork traps on the propagation of supercoiled monomers. The results presented here demonstrate a role of the fork trap in the repetitive cycle of circular DNA replication in an in vitro reconstituted system.

\section{Materials and Methods}

\subsection{Replication Cycle Reaction}

RCR was performed essentially as described previously [23]. A 10× RCR buffer ( $200 \mathrm{mM}$ Tris- $\mathrm{HCl}$ $\mathrm{pH}$ 8.0, 1.6 $\mathrm{M}$ potassium acetate, $100 \mathrm{mM} \mathrm{Mg}(\mathrm{OAc})_{2}, 40 \mathrm{mM}$ dithiothreitol (DTT), $40 \mathrm{mM}$ creatine phosphate, $10 \mathrm{mM}$ each NTP, $1 \mathrm{mM}$ each dNTP, $0.5 \mu \mathrm{g} / \mathrm{mL}$ yeast tRNA, $2.5 \mathrm{mM}$ NAD+, $100 \mathrm{mM}$ ammonium sulfate, and $1 \mathrm{mM}$ Tiron) and a $5 \times$ Enzyme mix $(2.5 \mathrm{mg} / \mathrm{mL}$ bovine serum albumin, $100 \mathrm{ng} / \mathrm{mL}$ creatine kinase, $0.5 \mathrm{mM}$ ATP, $2 \mu \mathrm{M} \mathrm{SSB}_{4}, 280 \mathrm{nM} \mathrm{IHF}_{2}, 1 \mu \mathrm{M}$ DnaG, $200 \mathrm{nM}$ DnaN $_{2}, 70 \mathrm{nM}$ Pol III* $100 \mathrm{nM} \mathrm{DnaB}_{6} \mathrm{C}_{6}, 500 \mathrm{nM}$ DnaA, $22 \mathrm{nM}$ RNaseH, $140 \mathrm{nM}$ ligase, $170 \mathrm{nM}$ Pol I, $250 \mathrm{nM}$ gyrase $\left(G_{r r A} B_{2}\right), 25 \mathrm{nM}$ Topo IV ( $\left.\operatorname{ParC}_{2} E_{2}\right), 200 \mathrm{nM}$ Topo III, and $200 \mathrm{nM}$ RecQ) were prepared. The $5 \mu \mathrm{L}$ reaction mixture was assembled on ice and included $10 \times \mathrm{RCR}$ buffer $(1 \mu \mathrm{L}), 5 \times$ Enzyme mix $(0.5 \mu \mathrm{L})$, and the indicated amount of Tus or Cre. After the addition of oriC circular DNA, the reaction was incubated at $30^{\circ} \mathrm{C}$ or $33^{\circ} \mathrm{C}$ for $3 \mathrm{~h}$, and then stopped by diluting 10 -fold with Stop buffer $(25 \mathrm{mM}$ Tris-HCl pH 8.0, $25 \mathrm{mM}$ EDTA, $0.1 \%$ sodium dodecyl sulfate, $0.05 \mathrm{mg} / \mathrm{mL}$ proteinase $\mathrm{K}, 5 \%$ glycerol, and $0.1 \%$ bromophenol blue). Preparation lot of $5 \times$ Enzyme mix used in Figure 1 was different from that in the other Figures, and the RCR activity was slightly different. An aliquot $(2 \mu \mathrm{L})$ was analyzed by $0.5 \%$ or $1.0 \%$ agarose gel electrophoresis, followed by SYBR Green I staining (Molecular Probes, Eugene, USA). The images were acquired with a Typhoon FLA 9500 scanner (GE Healthcare, 
Uppsala Sweden). The band intensity was quantified to calculate the ratio of concatemers to the sum of concatemers and supercoils. All experiments were performed at least twice, and a representative example is shown.

\subsection{Purified Proteins}

UvrD (82 kDa) was overproduced in BL21 (DE3) cells harboring pET-UvrD, which was constructed by cloning the $u v r D$ gene into the pET vector. The cells were grown to $\mathrm{OD}_{600}=0.75$ in LB medium containing $50 \mu \mathrm{g} / \mathrm{mL}$ carbenicillin, and then incubated for a further $2 \mathrm{~h}$ with $0.4 \mathrm{mM}$ IPTG. The cells were then lysed in Lysis buffer $(50 \mathrm{mM}$ Tris- $\mathrm{HCl}$ pH 8.0, 10\% sucrose, $480 \mathrm{mM} \mathrm{NaCl}, 10 \mathrm{mM}$ spermidine, $2 \mathrm{mM}$ DTT, $20 \mathrm{mM}$ EDTA, $0.25 \mathrm{mg} / \mathrm{mL}$ lysozyme, and $100 \mathrm{mM}$ PMSF). After removal of nucleic acids by precipitation with $0.25 \%$ polyethyleneimine, ammonium sulfate $(0.16 \mathrm{~g} / \mathrm{mL})$ was added to the lysate supernatant to precipitate the protein. The ammonium sulfate precipitate was resuspended in Column buffer (50 mM Tris- $\mathrm{HCl}$ pH 7.5, 20\% glycerol, $5 \mathrm{mM}$ DTT, and $1 \mathrm{mM}$ EDTA) and applied to a HiTrap Heparin column (GE Healthcare) equilibrated with Column buffer containing $100 \mathrm{mM} \mathrm{NaCl}$. After a wash step, the UvrD fractions were eluted with a gradient of 100-500 mM NaCl in Column buffer and pooled. Tus and other replication proteins were purified as described previously [23]. Cre recombinase was purchased from New England Biolabs (MA, USA).

\subsection{Plasmid Construction}

The pPKOZ plasmid $(8.9 \mathrm{~kb})$, which contains a $1 \mathrm{~kb}$ oriC cassette, a lacZ gene, and a kanamycin resistance gene, was constructed as described previously [23]. For construction of pPKOZ derivatives (pKZter_1-6, pKZter_5'ter, and pKZter_3'ter), a PKOZ fragment (8.9 kb) was PCR-amplified using pPKOZ as a template and primers SUE1254/SUE1255 targeting a region spanning $4.5 \mathrm{~kb}$ upstream and $3.4 \mathrm{~kb}$ downstream of the oriC cassette. A series of ter-cassettes, each containing ter $B$ sequences (5'-AGTATGTTGTAACTAAAG-3') and 20 bp overlapping ends for subsequent seamless ligation with the ends of the PKOZ fragment through an originally developed method (Recombination Assembly; to be published elsewhere), were PCR-amplified from pOriDif (12.3 kb) [23] with terB-containing primers. For pKZter_1 $(9.1 \mathrm{~kb})$, ter-cassette_1 $(0.2 \mathrm{~kb})$ was amplified with primers SUE1256_1/SUE1257_1. For pKZter_2 $(9.4 \mathrm{~kb})$, ter-cassette_2 $(0.5 \mathrm{~kb})$ was amplified with primers SUE1256_2/SUE1257_2. For pKZter_3 $(9.9 \mathrm{~kb})$, ter-cassette_3 $(1 \mathrm{~kb})$ was amplified with primers SUE1256_3/SUE1257_3. For pKZter_4 $(10.9 \mathrm{~kb})$, ter-cassette_4 $(2 \mathrm{~kb})$ was amplified with primers SUE1256_4/SUE1257_4. For pKZter_5 $(12.9 \mathrm{~kb})$, ter-cassette_5 $(4 \mathrm{~kb})$ was amplified with primers SUE1256_5/SUE1257_5. For pKZter_6 $(19.0 \mathrm{~kb})$, ter-cassette_6 (10 kb) was amplified with primers SUE1322/SUE1323. For pKZ_5'ter $(9.1 \mathrm{~kb})$, ter-cassette_5'ter $(0.2 \mathrm{~kb})$ was amplified with primers SUE1509/SUE1257_1. For pKZ_3'ter $(9.1 \mathrm{~kb})$, ter-cassette_3'ter $(0.2 \mathrm{~kb})$ was amplified with primers SUE1256_1/SUE1510. The ligation products were subjected to transformation of E. coli strain $\mathrm{DH} 5 \alpha$, and each oriC plasmid was recovered from a kanamycin-resistant colony.

For construction of pCLter30k, the Lter11 (a $14.8 \mathrm{~kb}$ chromosomal region from argS to folE) and Lter17 (a $14.8 \mathrm{~kb}$ chromosomal region from yeaI to $\mathrm{dmlA}$ ) fragments were PCR-amplified from E. coli strain $\Delta 33$ a [24] with primers Lter1f/Lter5r_2OL and SUE972/SUE973, respectively. The OL cassette (451 bp), which contained oriC sequences (286 bp) and loxP sequences (5'-ATAACTTCGTATAGCATACATTATACGAAGTTAT-3'), was prepared through polymerase cycling assembly [25] using oligo DNAs AO15, AO16-loxP, AO17, AO18, AO19, and AO20, with amplification primers LterOf/LterOr. To generate the Cm-OL fragment $(1.2 \mathrm{~kb})$, a chloramphenicol-resistant $(\mathrm{Cm})$ cassette $(0.75 \mathrm{~kb})$ was PCR-amplified from pACYC184 with primers LterCf_2/LterCr_2, and then ligated with the OL cassette through overlap PCR with amplification primers LterCf_2/SUE986. The LterCf_2 and SUE986 primers contained 60 bp overlapping ends for subsequent seamless ligation with the ends of the Lter11 and Lter17 fragments, respectively. The ter-cassette_L $(0.2 \mathrm{~kb})$ was PCR-amplified from pOriDif with primers SUE1700 and SUE1559, which contained the terB sequences and $40 \mathrm{bp}$ overlapping ends for subsequent seamless ligation with the ends of the Lter11 and 
Lter17 fragments, respectively. The four fragments (Lter11, Cm-OL, Lter17, and ter-cassette_L) were simultaneously assembled through Recombination Assembly. The assembly products were subjected to transformation of $E$. coli strain DH5 $\alpha$, and the pCLter30k plasmid ( $30.9 \mathrm{~kb})$ was recovered from a chloramphenicol-resistant colony.

For construction of pCLter_1 and pCLter_2, the OLDT cassette (424 bp) was constructed by PCR amplification of the OL cassette with terB-containing primers SUE1046/SUE1047. For the Cm-OLDT fragment $(1.2 \mathrm{~kb})$, the $\mathrm{Cm}$ cassette $(0.75 \mathrm{~kb})$ was ligated with the OLDT cassette through overlap PCR with primers LterCf_2/SUE1047. The DCW2 fragment $(1.1 \mathrm{~kb})$ and DCW6 fragment $(3.2 \mathrm{~kb})$, which consisted of a part of a region on the E. coli chromosome called the division and cell wall (dcw) cluster [26], were PCR-amplified from the MG1655 genome with primers SUE1158/SUE1161 and SUE1158/SUE1110, respectively. Subsequently, $60 \mathrm{bp}$ overlapping sequences were added to the Cm-OLDT fragment for seamless ligation with the DCW2 or DCW6 fragment, through PCR amplification with primers SUE1096/SUE1313 or SUE1096/SUE1314, respectively. The ligation products were subjected to transformation of E. coli strain DH5 $\alpha$, and pCLter_2 (a $2.3 \mathrm{~kb}$ plasmid consisting of Cm-OLDT and DCW2) and pCLter_1 (a $4.5 \mathrm{~kb}$ plasmid consisting of Cm-OLDT and DCW6) were recovered from chloramphenicol-resistant colonies.

For construction of pUC_OLDT and pUC_OriC300, the pUC fragment $(2.7 \mathrm{~kb})$ was PCR-amplified from pUC19 with primers SUE1156/SUE1361, which annealed to opposite sequences located immediately downstream of the lacZ $\alpha$ gene. The OLDT2 cassette $(544 \mathrm{bp})$ and oriC300 control cassette (481 bp), each containing $40 \mathrm{bp}$ overlapping ends for subsequent seamless ligation with the pUC fragment, were PCR-amplified with primers SUE1684/SUE1685 from the OLDT cassette or the $1 \mathrm{~kb}$ oriC cassette, respectively. The ligation products were subjected to transformation of E. coli strain DH5 $\alpha$, and pUC_OLDT (a $3.1 \mathrm{~kb}$ plasmid consisting of OLDT2 and pUC fragments) and pUC_OriC300 (a $3.0 \mathrm{~kb}$ plasmid consisting of oriC300 and pUC fragments) were recovered from ampicillin-resistant colonies.

The primer sequences are listed in Supplementary Table S1. All plasmids were purified using the QIAprep Miniprep Kit (Qiagen, Venlo, The Netherlands), and the ter sequences were verified by DNA sequencing.

\section{Results}

\subsection{Chromosome-Like Arrangement of oriC-ter Sites Is Detrimental to the RCR Propagation of Circular DNA}

In our previous studies of RCR, we found that propagation of supercoiled monomers of circular DNA is inhibited slightly by Tus-ter fork traps positioned opposite oriC, while the fork traps efficiently block the generation of concatemer byproducts due to rolling circle replication [23]. In RCR, the circular DNA has two inverted ter sites with the non-permissive sides facing each other (inward-facing), such that the arriving fork originating from oriC is permitted to pass through, but the fork leaving the termination region is blocked, thereby mimicking the oriC-ter arrangement on circular chromosomes. In the previous experiment, $\mathrm{RCR}$ was performed at $30^{\circ} \mathrm{C}$ for only $1 \mathrm{~h}$, during which the replication cycle repeats up to approximately seven times, as deduced from the doubling time of circular DNA (approximately $8 \mathrm{~min}$ ) [23]. When the incubation time was extended to $3 \mathrm{~h}$, inhibition of the propagation of target supercoiled monomers containing inward-facing ter sites (pKZter_1) became much more severe (Figure 1). In reactions using circular DNA lacking ter sites (pPKOZ), RCR efficiently propagated concatemers and supercoiled monomers, and no apparent inhibition by Tus was detected. These observations indicate that inward-facing ter sites positioned opposite oriC are detrimental to repetition of the replication cycle in RCR. 

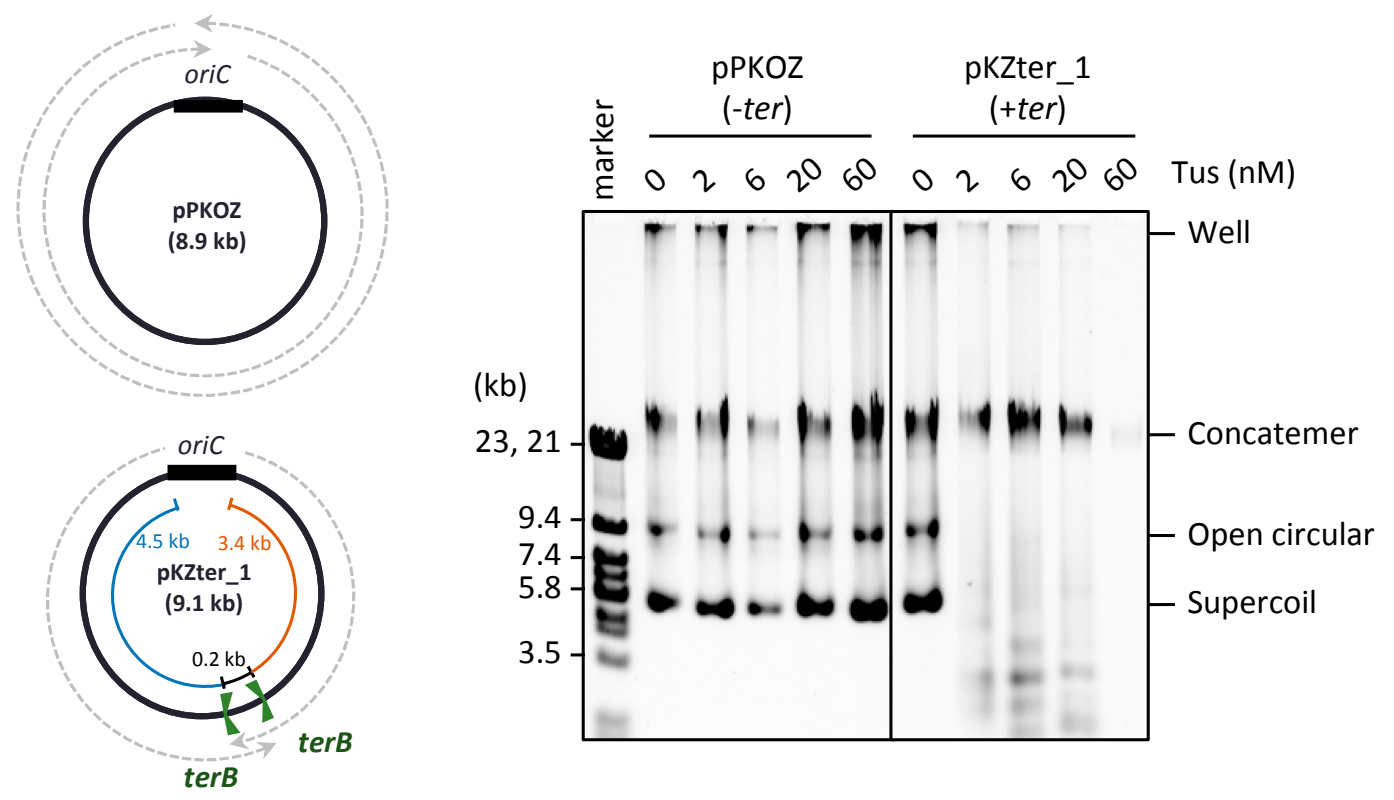

Figure 1. Effect of Tus on the RCR propagation of circular DNA in which the oriC-ter arrangement mimics the chromosome position. pKZter_1 (+ter) is a pKOZ (-ter) derivative containing inward-facing ter sites opposite oriC. The regions available for fork progression from the oriC cassette are indicated by dotted arrows on the circular map. pPKOZ or pKZter_1 (2.5 ng) was incubated in the RCR mixture at $30{ }^{\circ} \mathrm{C}$ for $3 \mathrm{~h}$ in the presence of the indicated concentrations of Tus. Aliquots $(0.2 \mu \mathrm{L})$ were analyzed by $0.5 \%$ TBE-agarose gel electrophoresis and SYBR Green I staining. The linear DNA size marker fragments were derived from lambda phage DNA (marker).

3.2. The Arrangement of ter Sites, Rather Than the Distance between Them, Is Critical for Repetition of the Replication Cycle

The minimum distance between the two inward-facing ter sites on the E. coli $4.6 \mathrm{Mb}$ chromosome is $267 \mathrm{~kb}$ (the distance between $\operatorname{ter} A$ and $\operatorname{ter} C$ ) [15]. On the other hand, the distance between the ter sites in the circular DNA we tested (pKZter_1) was much shorter $(0.2 \mathrm{~kb})$. Therefore, we examined whether a certain inter-ter distance is required for continuous repetition of the replication cycle in RCR. Five additional circular DNAs (pKZter_2-6), in which the distance between the two inward-facing ter sites was extended to $0.5,1,2,4$, or $10 \mathrm{~kb}$, were constructed by inserting various lengths of DNA into the inter-ter region. Propagation of the circular DNA was still inhibited by Tus when the inter-ter distance was extended to $4 \mathrm{~kb}$ (pKZter_5), but a $10 \mathrm{~kb}$ inter-ter distance (pKZter_6) allowed efficient propagation of supercoiled monomers, even in the presence of Tus (Figure 2A). Furthermore, Tus severely repressed the production of unwanted concatemers while allowing propagation of the supercoiled monomers of pKZter_6, indicating that the Tus-ter traps operated properly to repress rolling circle replication.

There are at least two possible explanations for how pKZter_6 was able to escape the inhibitory effect of Tus-ter traps on repetition of the replication cycle. The first is that the longer distance between oriC and the trap position for each bidirectional fork (14.5 and $13.4 \mathrm{~kb}$ for pKZter_6, in contrast to 8.5 and $7.4 \mathrm{~kb}$ for pKZter_5; see plasmid maps in Figure 2A) provides more time for the second fork to start from oriC before the first fork reaches the trap site. Unidirectional fork progression can take place in the early stage of replication in the in vitro oriC replication reaction [27]. Because the fork speed is approximately $0.5 \mathrm{~kb} / \mathrm{s}$ [28], the fork that started earlier would reach the trap site in pKZter_5 in approximately $15 \mathrm{~s}$ (based on a distance of $7.4 \mathrm{~kb}$ ). In this case, the situation in which one arm of the fork is trapped before the other arm leaves oriC may result in detrimental effects on replication of the replication cycle. To test this hypothesis, we constructed pCLter30k, a long arm version of pKZter_1 that had a $15 \mathrm{~kb}$ region between oriC and the non-permissively oriented ter site for each left and right arm, while the distance between the two inward-facing ter sites remained unchanged 
$(0.2 \mathrm{~kb})$. Unexpectedly, extension of the left and right arm distances did not overcome the inhibitory effect of Tus on RCR propagation (Figure 2B).

A
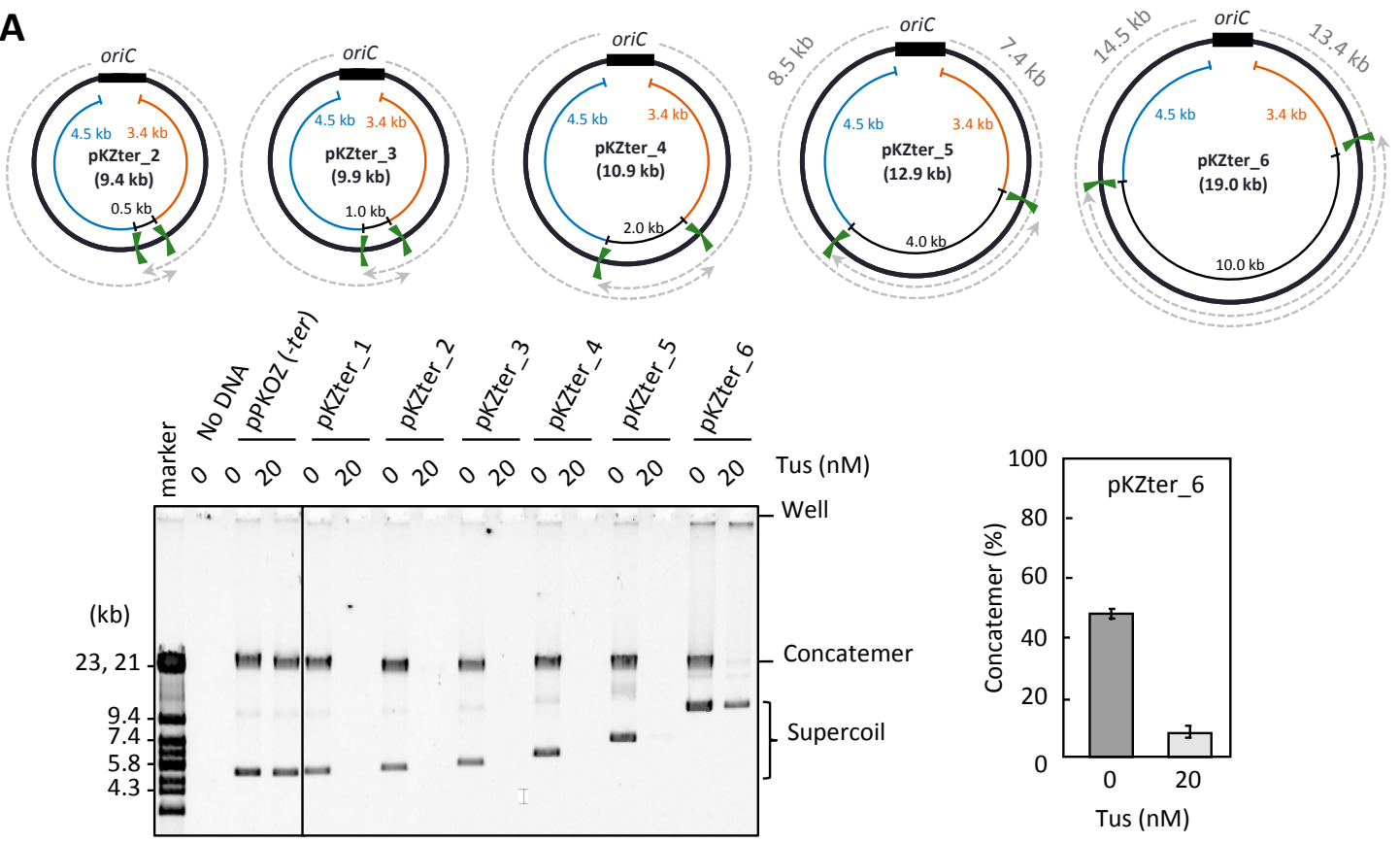

B
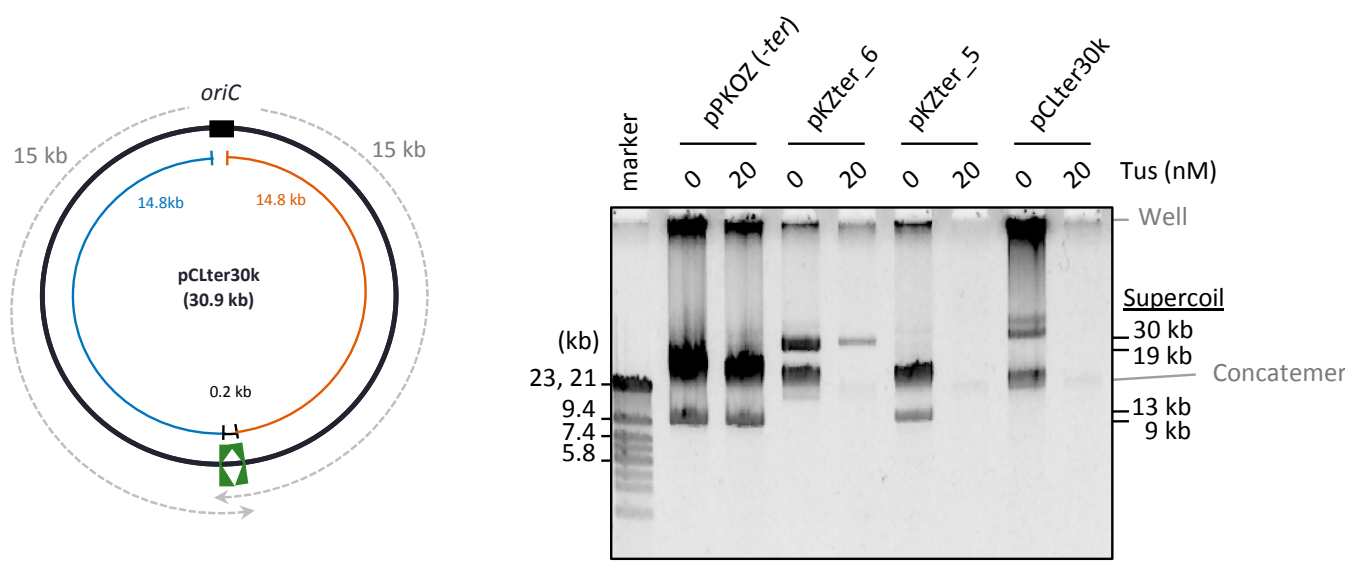

Figure 2. Effect of the inter-ter length on the RCR propagation in the presence of Tus. (A) pKZter_2-6 are pKZter_1 derivatives in which the length between the inward-facing ter sites was extended to $0.5,1.0,2.0,4.0$, or $10 \mathrm{~kb}$. The circular maps are shown as in Figure 1. The indicated plasmid (2.5 ng) was incubated in the RCR mixture at $30{ }^{\circ} \mathrm{C}$ for $3 \mathrm{~h}$ in the absence $(0 \mathrm{nM})$ or presence $(20 \mathrm{nM})$ of Tus. The product was analyzed by $0.5 \%$ TBE-agarose gel electrophoresis and SYBR Green I staining. The ratio of concatemers to the sum of concatemers and supercoils is shown in a graph as the average value of three repetitive experiments with standard deviation. (B) In pCLter30k, the regions available for fork progression were $15 \mathrm{~kb}$ (dotted arrows), while the inter-ter length was $0.2 \mathrm{~kb}$. RCR was performed as in (A), except that a $1 \%$ agarose gel was used to separate large supercoiled DNAs from concatemers. Large supercoiled DNA migrates more slowly than linear DNA [29]. DNA size marker fragments were derived from lambda phage DNA (marker).

The second possible explanation for how pKZter_6 was able to escape the inhibitory effect of Tus-ter traps on repetition of the replication cycle involves the arrangement of the two ter sites on the pKZter_6 circular map, in which each ter site is in close proximity to oriC rather than the site opposite oriC. The inter-ter distance thus occupies more than $50 \%$ of total plasmid size, in contrast to that of pKZter_5 (31\%). Such an arrangement may increase opportunities for the two bidirectional 
forks to meet and merge each other before being trapped at the ter site, while an unwanted portion of forks that escape from the fork merge event are trapped, thereby repressing the rolling circle replication. This second explanation was addressed using pCLter_1 and pCLter_2. These DNA constructs contained two ter sites flanking both sides of oriC, with an orientation permitting the forks to leave but not enter oriC. In addition, the distances between the two inward-facing ter sites in pCLter_1 and pCLter_2 (4.1 kb and $1.9 \mathrm{~kb}$, respectively) were as short as those in pKZter_5 and pKZter_4, respectively, the propagation of which was inhibited by Tus (see above). As shown in Figure 3, pCLter_1 and pCLter_2 were propagated as supercoiled monomers and concatemer production was suppressed in the presence of Tus, supporting the idea that the inhibition of supercoiled monomer propagation by Tus in the previous experiments was caused by fork merging through the Tus-ter trap. We also observed that, for the smaller-sized DNA, pCLter_2 $(2.3 \mathrm{~kb})$, production of the supercoiled monomers was inefficient in the absence of Tus, and rolling circle replication (i.e., concatemer production) was predominant. Propagation of the supercoiled monomers of pCLter_2 was enhanced by Tus, probably due to the fact that the repression of rolling circle replication can increase the amount of replication components available for the replication cycle mode of circular DNA.

Considering that fork merging through the Tus-ter trap is detrimental in RCR, we hypothesized that the detrimental effect would be detected even when the two ter sites positioned opposite oriC are oriented in the same direction. To test this possibility, we constructed pKZter_5'ter and pKZter_3'ter, pKZter_1 derivatives with two right- or left-oriented ter sites positioned opposite oriC and separated by $0.2 \mathrm{~kb}$ (Figure 4). As expected, in the presence of Tus, RCR propagation of pKZter_5'ter and pKZter_3'ter was inhibited, whereas the control pKZter_6 DNA was propagated as supercoiled monomers. When the contrast of the original image was increased, slight propagation products were detected in the reactions using pKZter_5'ter or pKZter_3'ter, but not in the reaction using pKZter_1-5 (Supplementary Figure S1). This result is reasonable considering that the chance of fork merging without mediating the Tus-ter trap could increase in the situation where the traps halt only a fork coming from one direction, compared with the situation where forks from both directions are halted.
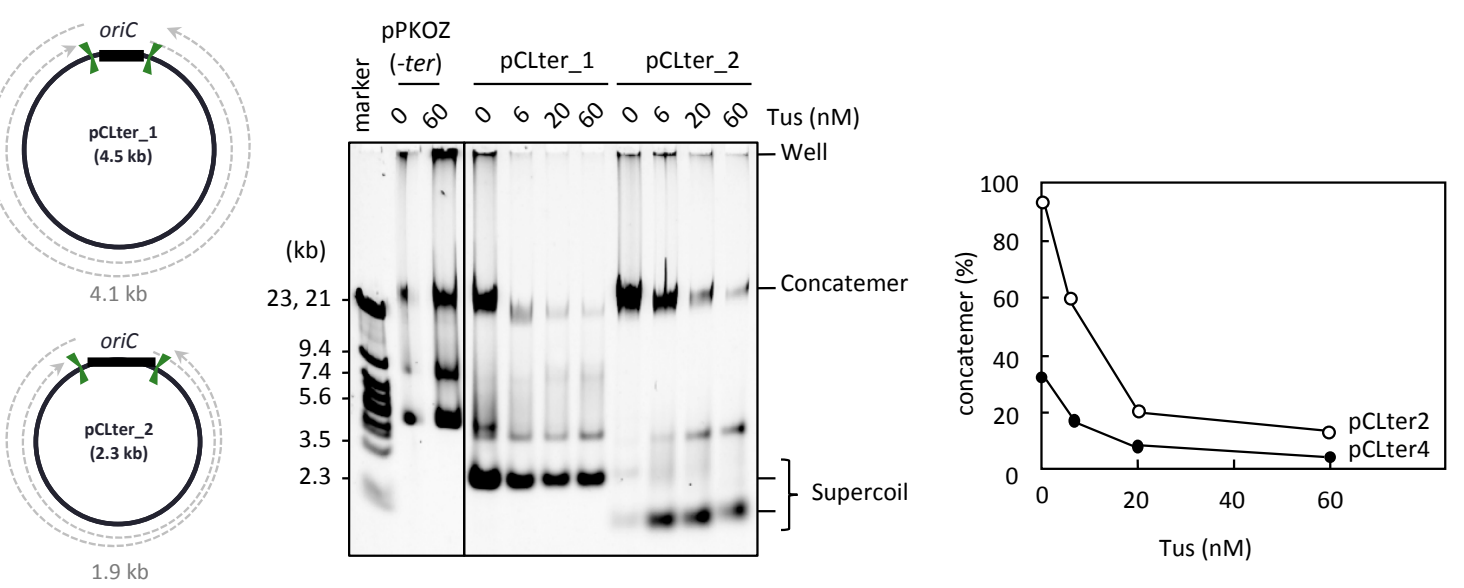

Figure 3. RCR propagation of circular DNA containing ter sites on both sides of oriC. The circular maps of pCLter_1 and pCLter_2 are shown as in Figure 1. The indicated plasmid (2.5 ng) was incubated in the RCR mixture at $30{ }^{\circ} \mathrm{C}$ for $3 \mathrm{~h}$ in the absence $(0 \mathrm{nM})$ or presence $(6,20$, or $60 \mathrm{nM})$ of Tus. The product was analyzed by $0.5 \%$ TBE-agarose gel electrophoresis and SYBR Green I staining. The ratio of concatemers to the sum of concatemers and supercoils is shown in a graph. 

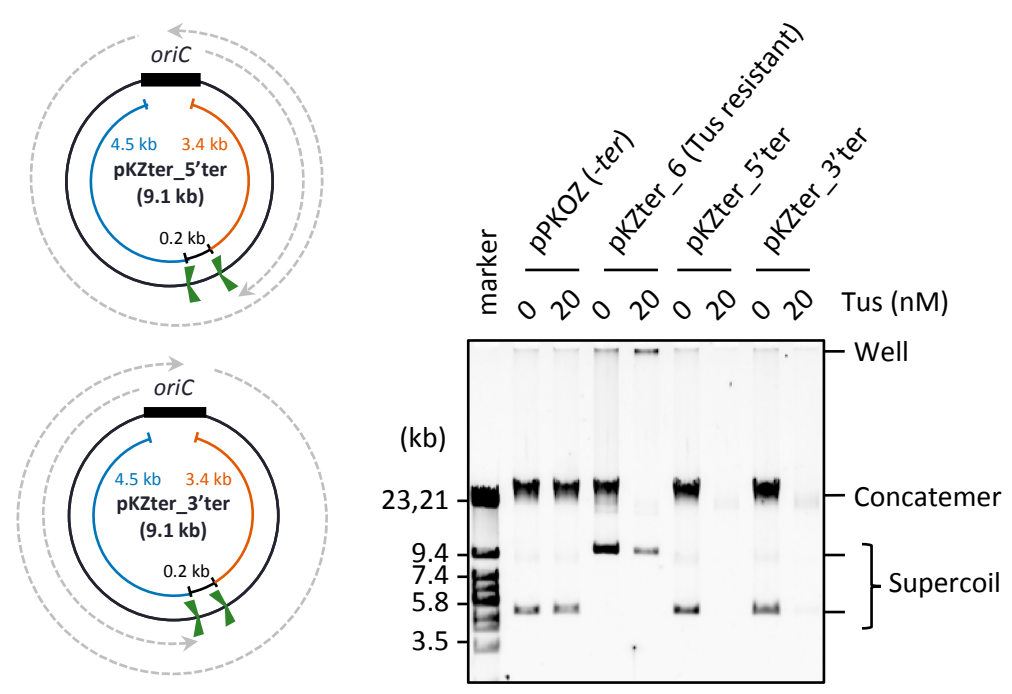

Figure 4. Effect of two same direction ter sites placed opposite oriC. The circular maps of pKZter_5'ter and pKZter_3'ter are shown as in Figure 1. The indicated plasmid (2.5 ng) was incubated in the RCR mixture at $30{ }^{\circ} \mathrm{C}$ for $3 \mathrm{~h}$ in the absence $(0 \mathrm{nM})$ or presence $(20 \mathrm{nM})$ of Tus. The product was analyzed by $0.5 \%$ TBE-agarose gel electrophoresis and SYBR Green I staining.

\subsection{UvrD Helicase Rescues the Detrimental Effect of the Tus-ter Traps in RCR}

A previous study showed that UvrD helicase is required for growth of cells whose left and right chromosome arms have ectopic ter sites in non-permissive orientation for the replication forks progressed from oriC, suggesting that $\mathrm{UvrD}$ may function in the removal of Tus from ter [29]. Therefore, we examined whether UvrD is able to rescue the detrimental effect of Tus on circular DNA propagation in RCR. We used pKZter_5, the propagation of which was inhibited by Tus due to two ter sites positioned closer to a region opposite oriC than to oriC (see above). UvrD enabled pKZter_5 to propagate as supercoiled monomers without producing concatemers, even in the presence of Tus (Figure 5). In a control reaction using pKZter_6, which propagated as supercoiled monomers in the presence of Tus, no marked effect of UvrD was observed. These results suggest that UvrD plays a role in elimination of the detrimental effect of Tus and allows the trapped fork to merge properly for the subsequent processes of the replication cycle.

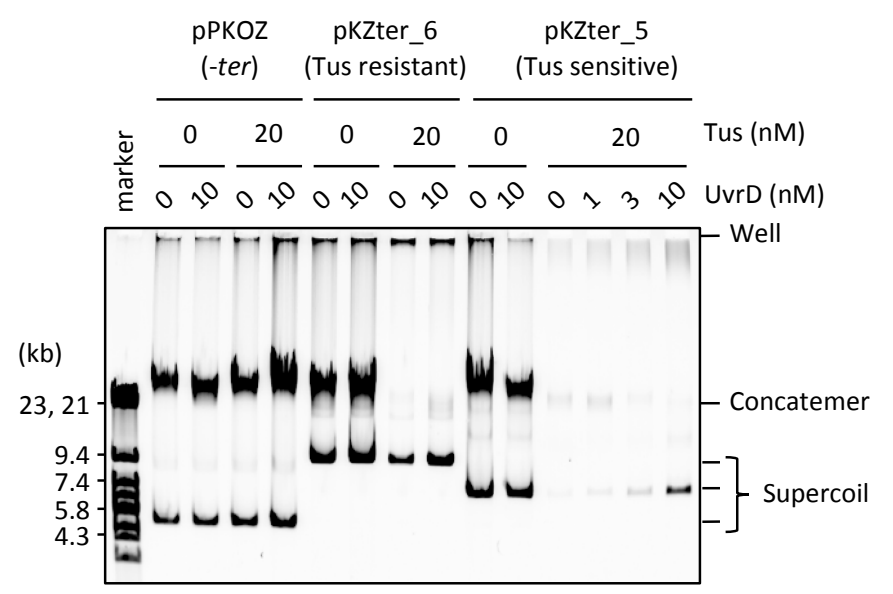

Figure 5. UvrD helicase rescues the detrimental effect of Tus in RCR. pPKOZ (-ter), pKZter_6 (Tus resistant), or pKZter_5 (Tus sensitive) was subjected to RCR propagation in the absence or presence of Tus as described in Figure 2A, except that the indicated concentration of UvrD was present in the reaction. 


\subsection{Resolution of the DNA Concatemers by the Cre-loxP System in RCR}

Next, we examined an alternative way to reduce concatemer production and enhance supercoiled DNA production in RCR. The Cre/loxP site-directed recombination system is an ideal system to resolve concatemer and generate circular DNAs through Cre-mediated recombination between directly repeating loxP sites [30]. We constructed pUC_OLDT (3 kb), which had a loxP site flanking oriC. When Cre recombinase was present in RCR, a ladder of bands representing supercoiled multimers was detected between the bands of concatemers and supercoiled monomers, along with a partial reduction of the concatemer products (pUC_OLDT; Figure 6). By contrast, there was no marked effect of Cre recombinase when the template circular DNA lacked the loxP site (pUC_OriC300; Figure 6). This result indicates that the Cre/loxP system can act in RCR to resolve concatemers that are generated due to defective termination of replication fork progression.
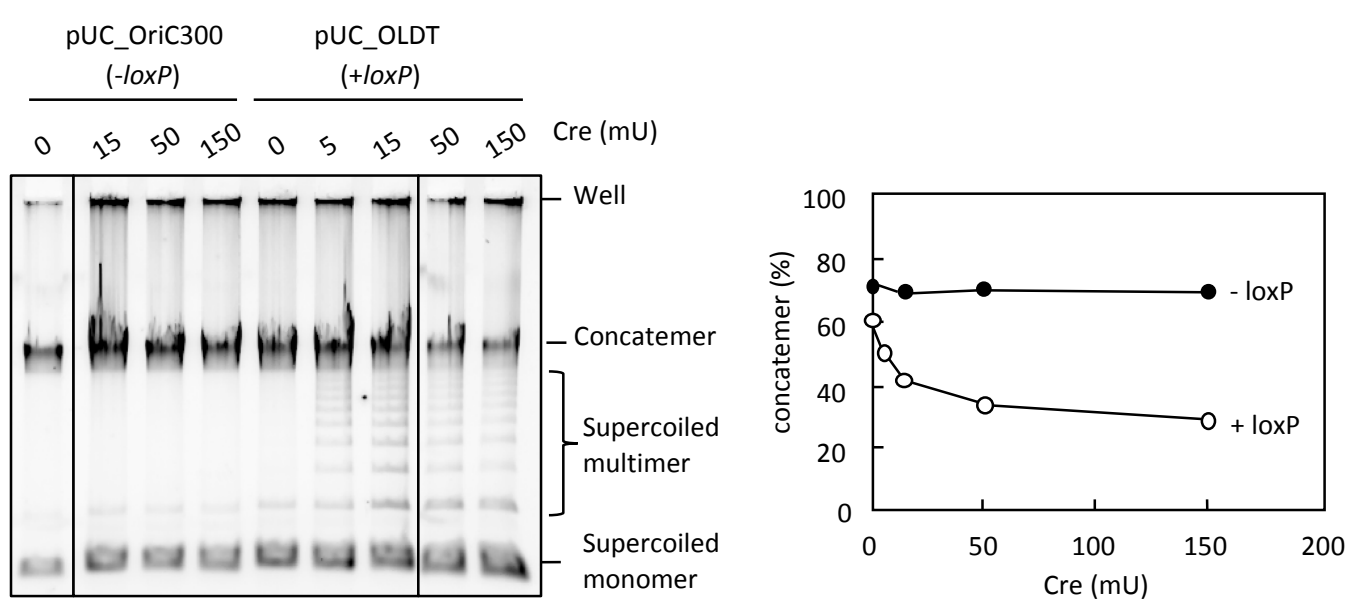

Figure 6. Effect of Cre-loxP system on the concatemer production in RCR. pUC_OriC300 (-loxP) or pUC_OLDT $(+\operatorname{lox} P)(0.05 \mathrm{ng})$ was incubated in the RCR mixture at $33^{\circ} \mathrm{C}$ for $3 \mathrm{~h}$ in the absence $(0 \mathrm{mU})$ or presence $(5,15,50$, or $150 \mathrm{mU})$ of Cre recombinase. The product was analyzed by $0.5 \%$ TBE-agarose gel electrophoresis and SYBR Green I staining. The ratio of concatemers to the sum of concatemers and supercoils is shown in a graph.

\section{Discussion}

In E. coli, chromosome replication proceeds bidirectionally from oriC and terminates at a region opposite oriC on the circular chromosome map through merging of the two converging replication forks. Multiple ter sites are located throughout the terminus half of the chromosome [15-17]. In this study, we found that Tus-ter fork traps located around the termination region are detrimental to repetition of the replication cycle of the oriC circular DNA in our in vitro reconstitution system, RCR. When the fork trap was repositioned to a region closer to oriC, allowing the forks on the left and right arms to pass through the terminus half of the circular map without being trapped by the Tus-ter system, supercoiled monomers were efficiently propagated and the production of unwanted concatemers was suppressed. These findings indicate that the fork merge termination event must occur without mediation of the Tus-ter system, in order to repeat the replication cycle in vitro, whereas the Tus-ter system acts to efficiently repress rolling circle-type over-replication. Our findings support the previous view that the replication termination can occur in the absence of the fork trap, and the fork trap serves as a safeguard system to halt the over-progression of the replication fork which might be disadvantageous because of the terminus-to-origin direction of replication [31]. In contrast to the effective position of the fork traps demonstrated in this in vitro study, the fork traps in E. coli and B. subtilis position near the termination region of their chromosome [31]. The direction of gene transcription in these chromosomes are majorly oriented in the origin-to-terminus direction [32]. The fork trap position of the chromosomes would be advantageous in the situation where replication and transcription occur simultaneously for avoiding 
head-on collision between the replication fork and the transcription machinery. Also, the cellular segregation process, in which several proteins, such as FtsK, Topoisomerase IV, and XerCD are involved, occurs via the dif site that is positioned at the terminus [31]. The chromosome arrangement of the fork trap can narrow the region for termination to coordinate with segregation.

Although intermediates leading to the concatemer formation in RCR is not presently clear, unidirectional fork progression and therefore nascent strand displacement by the fork might partly occur, leading to rolling circle replication. For unidirectional replication of the plasmid R1 in vivo, the rolling circle replication products have been observed, which is repressed in the presence of the Tus-ter system [33].

It is reported that the fork approaching the non-permissive face of ter is trapped by a mechanism in which Tus forms a stable locked complex with the fork structure [20]. The locked complex might impede the fork merge event or the decatenation event, resulting in inhibition of the subsequent replication cycle process. For example, the trapped fork might keep the locked complex state even upon a collision with a fork coming from the other direction until dissociation of the locked complex by mechanisms in which UvrD helicase is probably involved. Alternatively, the trapped fork might easily cause a double-strand break due to collision of the fork coming behind during repetition of the replication cycle. The resultant linear DNA is inactive for replication initiation in RCR [23].

In contrast to the in vitro observations, Tus-sensitive plasmids containing ter sites near the termination region (pKZter_1-5, pKZter_5' ter, and pKZter_3'ter) are replicated from oriC properly in vivo. These plasmids were stably inherited as oriC plasmids in E. coli DH5 $\alpha(t u s+)$ and were purified as well as the oriC DNAs lacking ter sites (data not shown). This observation implies that some mechanisms exist in vivo to overcome the detrimental effect of the fork trap. Indeed, we demonstrated in vitro that UvrD helicase rescues the inhibitory effect of the fork trap and allows repetition of the replication cycle in RCR (Figure 5). Consistent with this finding, UvrD is required for the growth of cells in vivo where replication forks are arrested at ectopic ter sites [29]. Whereas the $5^{\prime}$ to $3^{\prime}$ helicase activity of DnaB is halted by the Tus-ter trap, it has been demonstrated in vitro that the $3^{\prime}$ to $5^{\prime}$ helicase activity of UvrD is unaffected by this trap [34]. UvrD can initiate DNA unwinding from nicks in vitro [35]. It seems plausible that UvrD is loaded onto a leading strand gap when DnaB on the lagging strand of the fork encounters the fork trap, dissociating the Tus-ter locked complex, and thereby allowing the converging forks to merge properly.

When the size of the template circular DNA was relatively small (2.3 kb, pCLter_2), RCR propagation of the supercoiled monomers was inefficient, and concatemer production became predominant (Figure 3). Presumably, in the case of smaller circular DNAs, the fork merge event would occur less frequently because the fork that started earlier can complete replication of such a short template unidirectionally, without waiting for the other fork to start, resulting in rolling circle replication. We also observed that, when rolling circle replication was repressed by the Tus-ter trap, supercoiled monomer propagation of pCLter_2 became predominant.

In summary, we have established an efficient way to repress the unwanted concatemer byproducts in RCR by placing Tus-ter traps on either side of oriC, thereby enabling the supercoiled monomers to propagate predominantly and continuously. Propagation of DNA as individually separated molecules is a crucial event for self-replication of all living organisms. Our RCR system provides a powerful in vitro tool for reconstruction of self-replicating living systems.

Supplementary Materials: The following are available online at http://www.mdpi.com/2075-1729/8/4/43/s1, Table S1: Primer list. Figure S1: High-contrast image of Figures 2A and 4.

Author Contributions: M.S. conceived and designed the experiments; T.H., K.N. and S.H. performed experiments; M.S. and T.H. wrote the paper; all authors read and approved the final manuscript.

Funding: This study was supported by a grant from the Council for Science, Technology and Innovation (CSTI) ImPACT Program to M.S. 
Acknowledgments: We thank the technical staff in our laboratory (Rikkyo University) for assisting with protein purification, and Tatsuaki Kurata for plasmid construction. The E. coli strain $\Delta 33$ a was provided by the National Bio-Resource Project (NBRP) of MEXT, Japan.

Conflicts of Interest: The authors declare no conflict of interest.

\section{References}

1. Ichihashi, N.; Yomo, T. Constructive Approaches for Understanding the Origin of Self-Replication and Evolution. Life 2016, 6, 26. [CrossRef] [PubMed]

2. Forster, A.C.; Church, G.M. Towards synthesis of a minimal cell. Mol. Syst. Biol. 2006, 2, 45. [CrossRef] [PubMed]

3. Beattie, T.R.; Reyes-Lamothe, R. A Replisome's journey through the bacterial chromosome. Front. Microbiol. 2015, 6, 1-12. [CrossRef] [PubMed]

4. Kornberg, A.; Baker, T.A. DNA Replication, 2nd ed.; W.H. Freeman: New York, NY, USA, 1992.

5. Kaguni, J.M.; Kornberg, A. Replication initiated at the origin (oriC) of the E. coli chromosome reconstituted with purified enzymes. Cell 1984, 38, 183-190. [CrossRef]

6. Funnell, B.E.; Baker, T.A.; Kornberg, A. Complete enzymatic replication of plasmids containing the origin of the Escherichia coli chromosome. J. Biol. Chem. 1986, 261, 5616-5624. [PubMed]

7. Ozaki, S.; Katayama, T. DnaA structure, function, and dynamics in the initiation at the chromosomal origin. Plasmid 2009, 62, 71-82. [CrossRef] [PubMed]

8. Kaguni, J.M. Replication initiation at the Escherichia coli chromosomal origin. Curr. Opin. Chem. Biol. 2011, 15, 606-613. [CrossRef] [PubMed]

9. Johnson, A.; O'Donnell, M.M. Cellular DNA replicases: components and dynamics at the replication fork. Annu. Rev. Biochem. 2005, 74, 283-315. [CrossRef] [PubMed]

10. McHenry, C.S. DNA Replicases from a Bacterial Perspective. Annu. Rev. Biochem. 2011, 80, 403-436. [CrossRef] [PubMed]

11. Higuchi, K.; Katayama, T.; Iwai, S.; Hidaka, M.; Horiuchi, T.; Maki, H. Fate of DNA replication fork encountering a single DNA lesion during oriC plasmid DNA replication in vitro. Genes Cells 2003, 8, 437-449. [CrossRef] [PubMed]

12. Hiasa, H.; Marians, K.J. Initiation of bidirectional replication at the chromosomal origin is directed by the interaction between helicase and primase. J. Biol. Chem. 1999, 274, 27244-27248. [CrossRef] [PubMed]

13. Hiasa, H.; Marians, K.J. Primase couples leading- and lagging-strand DNA synthesis from oriC. J. Biol. Chem. 1994, 269, 6058-6063. [PubMed]

14. Hiasa, H.; Marians, K.J. Tus prevents overreplication of oriC plasmid DNA. J. Biol. Chem. 1994, 269, 26959-26968. [PubMed]

15. Neylon, C.; Kralicek, A.V.; Hill, T.M.; Dixon, N.E. Replication termination in Escherichia coli: structure and antihelicase activity of the Tus-ter complex. Microbiol. Mol. Biol. Rev. 2005, 69, 501-526. [CrossRef] [PubMed]

16. Hill, T.M.; Tecklenburg, M.L.; Pelletier, A.J.; Kuempel, P.L. tus, the trans-acting gene required for termination of DNA replication in Escherichia coli, encodes a DNA-binding protein. Proc. Natl. Acad. Sci. USA 1989, 86, 1593-1597. [CrossRef] [PubMed]

17. Kobayashi, T.; Hidaka, M.; Horiuchi, T. Evidence of a ter specific binding protein essential for the termination reaction of DNA replication in Escherichia coli. EMBO J. 1989, 8, 2435-2441. [CrossRef] [PubMed]

18. Hill, T.M.; Marians, K.J. Escherichia coli Tus protein acts to arrest the progression of DNA replication forks in vitro. Proc. Natl. Acad. Sci. USA 1990, 87, 2481-2485. [CrossRef] [PubMed]

19. Khatri, G.S.; MacAllister, T.; Sista, P.R.; Bastia, D. The replication terminator protein of E. coli is a DNA sequence-specific contra-helicase. Cell 1989, 59, 667-674. [CrossRef]

20. Mulcair, M.D.; Schaeffer, P.M.; Oakley, A.J.; Cross, H.F.; Neylon, C.; Hill, T.M.; Dixon, N.E. A Molecular Mousetrap Determines Polarity of Termination of DNA Replication in E. coli. Cell 2006, 125, 1309-1319. [CrossRef] [PubMed]

21. Lee, E.H.U.M.; Kornberg, A.; Hidakat, M.; Kobayashit, T.; Horiuchit, T. Escherichia coli replication termination protein impedes the action of helicases. Proc. Natl. Acad. Sci. USA 1989, 86, 9104-9108. [CrossRef] [PubMed] 
22. Roecklein, B.; Pelletier, A.; Kuempel, P. The tus gene of Escherichia coli: autoregulation, analysis of flanking sequences and identification of a complementary system in Salmonella typhimurium. Res. Microbiol. 1991, 142, 169-175. [CrossRef]

23. Su'etsugu, M.; Takada, H.; Katayama, T.; Tsujimoto, H. Exponential propagation of large circular DNA by reconstitution of a chromosome-replication cycle. Nucleic Acids Res. 2017, 45, 11525-11534. [CrossRef] [PubMed]

24. Iwadate, Y.; Honda, H.; Sato, H.; Hashimoto, M.; Kato, J. Oxidative stress sensitivity of engineered Escherichia coli cells with a reduced genome. FEMS Microbiol. Lett. 2011, 322, 25-33. [CrossRef] [PubMed]

25. Heyneker, H.L. Single-step assembly of a gene and entire plasmid from large numbers of oligodeoxyribonucleotides. Genes 1995, 164, 49-53.

26. Vicente, M.; Gomez, M.J.; Ayala, J.A. Regulation of transcription of cell division genes in the Eschericia coli dcw cluster. Cell. Mol. Life Sci. 1998, 54, 317-324. [CrossRef] [PubMed]

27. Smelkova, N.; Marians, K.J. Timely Release of Both Replication Forks from oriC Requires Modulation of Origin Topology. J. Biol. Chem. 2001, 276, 39186-39191. [CrossRef] [PubMed]

28. Tanner, N.A.; Loparo, J.J.; Hamdan, S.M.; Jergic, S.; Dixon, N.E.; van Oijen, A.M. Real-time single-molecule observation of rolling-circle DNA replication. Nucleic Acids Res. 2009, 37, 2-7. [CrossRef] [PubMed]

29. Bidnenko, V.; Lestini, R.; Michel, B. The Escherichia coli UvrD helicase is essential for Tus removal during recombination-dependent replication restart from Ter sites. Mol. Microbiol. 2006, 62, 382-396. [CrossRef] [PubMed]

30. Ghosh, K.; Van Duyne, G.D. Cre-loxP biochemistry. Methods 2002, 28, 374-383. [CrossRef]

31. Duggin, I.G.; Wake, R.G.; Bell, S.D.; Hill, T.M. The replication fork trap and termination of chromosome replication. Mol. Microbiol. 2008, 70, 1323-1333. [CrossRef] [PubMed]

32. Rocha, E.P.C.; Danchin, A. Gene essentiality determines chromosome organisation in bacteria. Nucleic Acids Res. 2003, 31, 6570-6577. [CrossRef] [PubMed]

33. Krabbe, M.; Zabielski, J.; Bernander, R.; Nordström, K. Inactivation of the replication-termination system affects the replication mode and causes unstable maintenance of plasmid R1. Mol. Microbiol. 1997, 24, 723-735. [CrossRef] [PubMed]

34. Hiasa, H.; Marians, K.J. Differential inhibition of the DNA translocation and DNA unwinding activities of DNA helicases by the Escherichia coli tus protein. J. Biol. Chem. 1992, 267, 11379-11385. [PubMed]

35. Runyon, G.T.; Bear, D.G.; Lohman, T.M. Escherichia coli helicase II (UvrD) protein initiates DNA unwinding at nicks and blunt ends. Proc. Natl. Acad. Sci. USA 1990, 87, 6383-6387. [CrossRef] [PubMed] 